\title{
Reference Renal Artery Diameter Is a Stronger Predictor of Contrast-Induced Nephropathy than Chronic Kidney Disease in Patients with High Cardiovascular Risk
}

\author{
Luca Zanoli ${ }^{a}$ d Stefania Rastelli ${ }^{a}$ Carmelita Marcantoni ${ }^{c}$ \\ Julien Blanco ${ }^{a}$ Davide Capodanno ${ }^{\mathrm{b}}$ Corrado Tamburino $^{\mathrm{b}}$ \\ Pietro Castellino ${ }^{a}$
}

Departments of a Internal Medicine and ${ }^{b}$ Cardiology, University of Catania, ${ }^{c}$ Division of Nephrology,

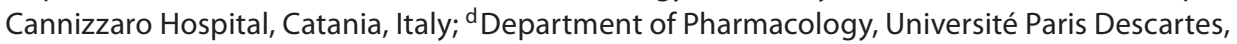
Hôpital Européen Georges Pompidou, Assistance Publique - Hôpitaux de Paris, INSERM U970, Paris, France

\section{Key Words}

Acute renal failure $\cdot$ Contrast media $\cdot$ lodixanol $\cdot$ RAS-CAD $\cdot$ Reference vessel diameter $\cdot$ Renal artery stenosis

\begin{abstract}
Introduction: The incidence of contrast-induced nephropathy (CIN) increases in high cardiovascular risk patients. Chronic kidney disease (CKD) is a known risk factor for CIN development. In a previous report, we demonstrated that the mean reference renal artery diameter (RVD) is an important determinant of CKD in patients undergoing coronary angiography for ischemic heart disease. However, RVD was never tested as a predictor of CIN. Aim: To look the predictors of CIN. Methods: A total of 218 consecutive patients undergoing coronary and renal angiography were enrolled from the cohort of the RAS-CAD study (NCT 01173666). CIN was defined as a relative increase in baseline serum creatinine $\geq 25 \%$ within 1 week of contrast administration. $\boldsymbol{R e}$ sults: The incidence of CIN was $22 \%$. In a fully adjusted model, contrast medium dose ( $20 \mathrm{ml}$ increase, OR 1.12, 95\% Cl 1.06-1.19, $\mathrm{p}<0.001$ ), iso-osmolar contrast media (OR 0.28, 95\% Cl 0.09-0.99, $\mathrm{p}<0.05$ ), atherosclerotic renovascular disease (OR 2.69, 95\% Cl 1.32-5.48, $\mathrm{p}<0.05$ ), and RVD $\left(1 \mathrm{~mm} / 1.73 \mathrm{~m}^{2}\right.$ increase, OR $\left.0.59,95 \% \mathrm{Cl} 0.41-0.86, \mathrm{p}<0.05\right)$ had the greatest effect on outcome
\end{abstract}

The present work was performed at the Department of Cardiology, University of Catania, Italy. 
and were identified as independent predictors of CIN. CKD was selected as a predictor of CIN only in a model without RVD. Conclusions: In patients undergoing coronary angiography for ischemic heart disease, RVD is a stronger predictor of CIN than CKD.

Copyright $\odot 2011$ S. Karger AG, Basel

\section{Introduction}

Contrast-induced nephropathy (CIN) is a common and serious complication of contrast media (CM) used in imaging studies and is the third leading cause of acute kidney injury in hospitalized patients [1]. The incidence of CIN is low in patients without cardiovascular risk factors [2] and increases in patients with high cardiovascular risk [3]. Patients who develop CIN after percutaneous coronary intervention are at an increased risk of both short- and long-term mortality $[3,4]$. Therefore, it is important to identify high-risk patients to reduce the incidence of CIN.

Many individual risk factors for the development of CIN have been detected. Chronic kidney disease (CKD) is one of the most important risk factors for CIN. However, the mean reference renal artery diameter (RVD) was never tested prospectively to predict CIN. In patients undergoing coronary angiography for ischemic heart disease, we detected that RVD and minimal luminal diameter (MLD) are stronger determinants of CKD than the severity of renal artery stenosis [5]. We hypothesized that RVD could be a predictor of CIN, independently of major confounders. We took advantage of a very well phenotyped high cardiovascular risk population (the RAS-CAD study [6, 7]; NCT 01173666) to investigate these points.

Our aim was to look at the predictors of CIN in patients undergoing coronary angiography for suspected coronary heart disease.

\section{Methods}

\section{Study Population}

The study conformed to the ethical guidelines of our institution, and informed consent was obtained from each participant. The primary outcome was the development of CIN, defined as a relative increase in baseline serum creatinine ( $\mathrm{sCr}$ ) $>25 \%$ within 1 week of contrast injection.

Patients were considered to be eligible for the study if they (1) were older than 18 years of age, (2) were referred for coronary angiography for ischemic heart disease, and (3) presented a stable baseline sCr concentration. Criteria for exclusion were (1) intravascular administration of an iodinated contrast medium (CM) within the previous month, (2) acute myocardial infarction, (3) pre-existing end-stage renal disease requiring dialysis, and (4) shock.

The patients' age, gender, and sCr value were used to determine the estimated glomerular filtration rate (GFR) according to the formula developed and validated using data obtained from patients of the Modification of Diet in Renal Disease study [8]: GFR $\left(\mathrm{ml} / \mathrm{min} / 1.73 \mathrm{~m}^{2}\right)=$ $186 \times(\mathrm{sCr})^{1.154} \times$ age $^{0.203} \times 0.742$ (if female) $\times 1.21$ (if Black). We considered a GFR $<60$ $\mathrm{ml} / \mathrm{min} / 1.73 \mathrm{~m}^{2}$ as CKD.

\section{CIN Prevention Protocol}

All patients were hydrated before angiography according to the ejection fraction (EF). Patients with $\mathrm{EF}>30 \%$ received $1 \mathrm{ml} / \mathrm{kg} / \mathrm{h}$ of saline intravenously starting $24 \mathrm{~h}$ before and ending $24 \mathrm{~h}$ after the coronary angiography. Patients with $\mathrm{EF} \leq 30 \%$ received $0.5 \mathrm{ml} / \mathrm{kg} / \mathrm{h}$ of 
saline intravenously $24 \mathrm{~h}$ before until $24 \mathrm{~h}$ after the coronary angiography. All patients with $\mathrm{sCr}>1.2 \mathrm{mg} / \mathrm{dl}$ received $\mathrm{N}$-acetylcysteine $(1,200 \mathrm{mg})$ twice daily starting $24 \mathrm{~h}$ before and ending $24 \mathrm{~h}$ after the coronary angiography.

$\mathrm{sCr}$ was measured within $24 \mathrm{~h}$ prior to angiography and during the first week after the administration of CM. The total volume of injected CM was recorded. The iso-osmolar CM (IOCM) iodixanol and the low-osmolar CM (LOCM) iobitridol, iopromide, iohexol, and iomeprol were used in this analysis. In this report, we look at the group effect of LOCM on CIN development.

\section{Renal Angiography}

To define the patency of the renal arteries, selective renal arteriography was performed at the end of the cardiac catheterization in the anterior-posterior and oblique projection with a hand injection of $4-8 \mathrm{ml}$ of CM. After calibration with the outer diameter of the contrastfilled catheter was performed to provide a standard, the size of the renal arteries was measured. In stenotic vessels, RVD was measured before the stenosis or, when unavailable, after the post-stenotic dilatation. Two qualified observers blinded to the patients' clinical information reviewed the angiographies. Each renal artery patency was measured twice. The average of the two operations was recorded. The mean of the left and right RVD was used in this analysis. Atherosclerotic renovascular disease (ARVD) was defined as the presence of angiographically evident renal artery stenosis.

\section{Statistical Analyses}

Statistical analyses were performed with SPSS 16 software (SPSS Inc., Chicago, Ill., USA). Continuous variables were reported using means and standard deviations. Categorical variables were described as counts and percentages. The clinical characteristics of patients with and without CIN were compared using analyses of variance for continuous variables and the $\chi^{2}$ test for categorical variables. Multivariate logistic regression analysis was performed to identify independent predictors of CIN and to estimate odds ratios (OR). Risk factors that were significant in the univariate analysis $(\mathrm{p} \leq 0.20)$ were available for selection in the final model; a backward stepwise method was used to select the best subset of independent predictors of CIN to avoid overfitting of the data. Three separate regression models were developed (model 1, 2 and 3, respectively). The set of variables tested in model 1 included all the univariate predictors of CIN; the set of variables tested in model 2 included all the univariate predictors of CIN tested in model 1 with the exception of RVD, and in model 3, we forced the CKD into the model.

\section{Results}

\section{Patients}

During the 6-month enrollment period, we consecutively enrolled 218 patients undergoing coronary angiography. Overall, the mean age of the patients was $64.2 \pm 10.6$ years, $74 \%$ were males, and baseline sCr was $1.07 \pm 0.31 \mathrm{mg} / \mathrm{dl}$. CKD was present in 57 patients (26\%); the incidence of CIN was $22 \%$. A total of 15 variables were associated ( $p \leq 0.20$ ) with the development of CIN and were selected to be studied in multivariate analysis. The selected correlates included demographics (age and female gender), clinical characteristics (pulse pressure, CKD, ARVD, RVD, hypertension, and smoking), drug therapy ( $\beta$-blockers, ACE inhibitors/sartans, and nitrates), and several angiographic and/or procedural characteristics (coronary artery disease, IOCM administration, and CM dose). Baseline characteristics and univariate analysis for CIN are reported in table 1. 
Table 1. Demographic and clinical data

\begin{tabular}{|c|c|c|c|c|}
\hline & $\begin{array}{l}\text { Whole group } \\
(\mathrm{n}=218)\end{array}$ & $\begin{array}{l}\text { CIN } \\
(n=48)\end{array}$ & $\begin{array}{l}\text { Non-CIN } \\
(\mathrm{n}=170)\end{array}$ & p value ${ }^{1}$ \\
\hline Age, years & $64 \pm 11$ & $68 \pm 11$ & $63 \pm 10$ & $<0.05$ \\
\hline Male sex & 74 & 65 & 76 & 0.15 \\
\hline Weight, kg & $75.8 \pm 13.5$ & $75.2 \pm 14.9$ & $75.9 \pm 13.1$ & 0.76 \\
\hline Height, m & $1.65 \pm 0.08$ & $1.64 \pm 0.07$ & $1.65 \pm 0.08$ & 0.52 \\
\hline Body mass index & $27.8 \pm 4.2$ & $27.8 \pm 4.7$ & $27.8 \pm 4.0$ & 0.99 \\
\hline Body surface area, $\mathrm{m}^{2}$ & $1.88 \pm 0.19$ & $1.87 \pm 0.20$ & $1.88 \pm 0.19$ & 0.65 \\
\hline Systolic blood pressure, $\mathrm{mm} \mathrm{Hg}$ & $131 \pm 195$ & $133 \pm 23$ & $131 \pm 17$ & 0.62 \\
\hline Diastolic blood pressure, $\mathrm{mm} \mathrm{Hg}$ & $78 \pm 11$ & $77 \pm 11$ & $79 \pm 12$ & 0.38 \\
\hline Pulse pressure, $\mathrm{mm} \mathrm{Hg}$ & $53 \pm 15$ & $56 \pm 16$ & $52 \pm 15$ & 0.20 \\
\hline Baseline sCr, mg/dl & $1.07 \pm 0.31$ & $1.12 \pm 0.41$ & $1.06 \pm 0.27$ & 0.38 \\
\hline $\mathrm{GFR}, \mathrm{ml} / \mathrm{min} / 1.73 \mathrm{~m}^{2}$ & $75 \pm 23$ & $74 \pm 31$ & $75 \pm 20$ & 0.81 \\
\hline CKD & 26 & 38 & 23 & $<0.05$ \\
\hline Dyslipidemia & 77 & 75 & 78 & 0.70 \\
\hline Smoking & 66 & 52 & 69 & $<0.05$ \\
\hline Diabetes & 38 & 44 & 37 & 0.36 \\
\hline Hypertension & 84 & 92 & 81 & 0.08 \\
\hline Stroke & 10 & 11 & 9 & 0.80 \\
\hline Peripheral vascular disease & 24 & 27 & 23 & 0.55 \\
\hline \multicolumn{5}{|l|}{ Therapy } \\
\hline Statins & 53 & 60 & 52 & 0.35 \\
\hline Diuretics & 28 & 31 & 27 & 0.64 \\
\hline$\beta$-Blockers & 40 & 52 & 36 & 0.06 \\
\hline ACE inhibitors/sartans & 54 & 64 & 52 & 0.14 \\
\hline Calcium antagonists & 19 & 17 & 19 & 0.69 \\
\hline$\alpha$-Blockers & 6 & 5 & 6 & 0.75 \\
\hline Nitrates & 34 & 48 & 30 & $<0.05$ \\
\hline Coronary artery disease involved & & & & $<0.05$ \\
\hline None & 9 & 4 & 11 & \\
\hline 1 vessel & 33 & 23 & 36 & \\
\hline 2 vessels & 23 & 23 & 24 & \\
\hline 3 vessels & 34 & 50 & 30 & \\
\hline ARVD & 35 & 50 & 31 & $<0.05$ \\
\hline $\mathrm{RVD}, \mathrm{mm} / 1.73 \mathrm{~m}^{2}$ & $5.48 \pm 0.97$ & $5.24 \pm 0.98$ & $5.57 \pm 0.97$ & $<0.05$ \\
\hline $\mathrm{CM}$ dose, $\mathrm{ml}$ & $202 \pm 132$ & $265 \pm 175$ & $185 \pm 112$ & $<0.05$ \\
\hline IOCM & 17 & 10 & 18 & 0.20 \\
\hline Hydration, $0.5 \mathrm{ml} / \mathrm{kg} / \mathrm{h}$ & 8 & 13 & 7 & 0.16 \\
\hline $\mathrm{N}$-acetylcysteine & 30 & 40 & 28 & 0.11 \\
\hline
\end{tabular}

Values are means $\pm \mathrm{SD}$ or percentages. ${ }^{1} \mathrm{CIN}$ vs. non-CIN.

\section{Multivariate Analyses}

Taking advantage of a logistic regression analysis for CIN development, the influence of various correlates on the outcome was evaluated. CM dose, IOCM, ARVD, and RVD had the greatest effect on outcome and were identified as independent predictors of CIN (model 1; table 2). When we developed a model excluding RVD from the set of variables studied by multivariate analysis, CKD was selected as an independent predictor of CIN (model 2; table 2). Therefore, we forced the variable CKD into the multivariate model (model 3; table 2). In the latter model, CKD did not reach a significant level (OR 1.49, 95\% CI 0.67-3.31, p = 0.33). The area under the ROC curve for model 1,2 and 3 was 0.74 (95\% CI $0.66-0.81, \mathrm{p}<0.001), 0.71$ (95\% CI 0.62-0.79, p < 0.001), and 0.74 (95\% CI 0.66-0.81, p < 0.001), respectively. 
Table 2. Multivariate logistic regression analyses for CIN

\begin{tabular}{lccc}
\hline & OR & $95 \%$ CI & p value \\
\hline Model 1 & & & \\
CM dose $(20 \mathrm{ml})$ & 1.12 & $1.06-1.19$ & $<0.001$ \\
IOCM & 0.28 & $0.09-0.99$ & $<0.05$ \\
ARVD & 2.69 & $1.32-5.48$ & $<0.05$ \\
RVD $\left(1 \mathrm{~mm} / 1.73 \mathrm{~m}^{2}\right)$ & 0.59 & $0.41-0.86$ & $<0.05$ \\
Model 2 & & & \\
CM dose $(20 \mathrm{ml})$ & 1.11 & $1.05-1.17$ & $<0.001$ \\
IOCM & 0.32 & $0.11-0.99$ & $<0.05$ \\
ARVD & 2.23 & $1.09-4.55$ & $<0.05$ \\
CKD & 1.95 & $0.91-4.16$ & 0.09 \\
Model 3 & & & \\
CM dose $(20 \mathrm{ml})$ & 1.12 & $1.06-1.19$ & $<0.001$ \\
IOCM & 0.28 & $0.09-0.91$ & $<0.05$ \\
ARVD & 2.47 & $1.19-5.14$ & $<0.05$ \\
RVD $\left(1 \mathrm{~mm} / 1.73 \mathrm{~m}^{2}\right)$ & 0.62 & $0.43-0.91$ & $<0.05$ \\
CKD & 1.50 & $0.68-3.33$ & 0.32 \\
\hline
\end{tabular}

\section{Discussion}

For the first time, RVD, a factor associated with CKD [5], has been examined as a risk factor for CIN. RVD was a stronger predictor of CIN than CKD in patients at high cardiovascular risk undergoing coronary angiography for ischemic heart disease.

For the first time, our data provide evidence that RVD is a better predictor of CIN than CKD. In our study, prevalence of CKD was significantly higher in patients who developed CIN than in those who did not. Interestingly, when tested together with RVD, CKD was not selected as a predictor of CIN. CM dose, IOCM, ARVD, and RVD were the most important factors associated with CIN (model 1; table 2). Due to its known impact on the development of CIN, we tested CKD as a possible predictor of CIN. When forced into the multivariate model (model 3; table 2), CKD does not reach a significant level $(\mathrm{p}=0.32)$. Therefore, we developed a model to test CKD as a determinant of CIN excluding RVD from the set of variables studied with multivariate analysis (model 2; table 2). As expected, in model 2, CKD was selected as a predictor of CIN. Therefore, our analysis confirmed that CKD is a predictor of CIN but led to the conclusion that RVD is a better predictor of CIN than CKD in patients at high cardiovascular risk.

CIN is classically attributed to vasoconstriction after iodinated CM injection [2, 9]. There is evidence that IOCM reduced the risk of CIN when compared to LOCM [10]. Our data confirm that the use of the IOCM iodixanol is associated with a reduced risk of CIN compared to LOCM. Moreover, our results confirm that the risk of CIN increases depending on CM dose, suggesting that coronary angiography in high-risk patients should be designed so that excessive volumes of CM are avoided; thus, the IOCM iodixanol should be preferred.

RVD could be related to GFR by several mechanisms. In case of stenosis, MLD could represent the limiting factor for kidney blood flow, while in the absence of stenosis, RVD could be determined by blood flow through flow-mediated dilation. The reduction of RVD may be the consequence of a decreased renal blood flow. Arterial diameter is dependent on several dynamic factors, among them the level of vascular tone on the short term and vascular remodeling on the long term. It is well known that long-term changes in local hemodynamic forces induce remodeling of arterial wall tissue, which is critical to vascular adapta- 
tion in the adult and to the progression of cardiovascular disease [11]. Sustained reductions in blood flow lead to flow-induced decreases in shear stress and inward arterial remodeling with a narrowing of lumen diameter [12]. Clinically significant arterial occlusive disease depressurizes the arterial tree downstream from the occlusion site and reduces the wall shear stress. Arterial remodeling limits change in wall shear stress [13].

In a previous study [5], we detected that in patients with low RVD or low MLD the OR for CKD was markedly elevated. The association between RVD and CKD was confirmed in a fully adjusted model. In the present study, we extended the predictive power of RVD prospectively and reported that RVD is an independent predictor of renal function impairment after CM administration in high cardiovascular risk patients.

To our knowledge, this is the first time that RVD was tested in a model for CIN including ARVD. We detected that CIN incidence is increased in patients with ARVD and that a reduction of mRAC predisposes to a higher incidence of CIN. The clinical implication of these data is the promising role of renal artery patency as a predictor of GFR reduction after $\mathrm{CM}$ administration. Further analyses are needed to examine the etiology of CIN.

Our CIN prevention protocol, developed according to patients' EF and baseline sCr, consisted in hydration and $\mathrm{N}$-acetylcysteine. Several studies identified volume depletion as a risk factor for the development of CIN; as a result, fluid administration is frequently recommended $[13,14]$. However, patients with low EF are more prone to congestive cardiac failure than patients with normal EF. In these patients, an over-hydration may dramatically increase the risk for heart failure. Therefore, we reduced the hydration in patients with $\mathrm{EF} \leq 30 \%$. Nacetylcysteine, a free radical scavenger with reported vasodilatory effects, has been reported in trials and in a recent meta-analysis to prevent CIN $[15,16]$. A 3-fold reduction in the incidence of CIN was reported in CKD patients undergoing elective coronary angiography following the administration of oral $\mathrm{N}$-acetylcysteine $(600 \mathrm{mg})$ in addition to intravenous isotonic saline [15]. Recent studies suggest that higher doses of $\mathrm{N}$-acetylcysteine may provide a significant protection against CIN: treatment with $1,200 \mathrm{mg} \mathrm{N}$-acetylcysteine given orally twice daily on the day before and the day of contrast exposure was associated with a lower risk of CIN compared with $600 \mathrm{mg} \mathrm{N}$-acetylcysteine [17]. In addition, $\mathrm{N}$-acetylcysteine side effects and drug interactions are very rare with continued use and are highly unlikely to result from the limited use for renal protection. Therefore, we used $\mathrm{N}$-acetylcysteine $(1,200 \mathrm{mg}$ twice daily) in patients at higher risk of CIN.

The major limitation of this study was that the renal angiography was performed without $3 \mathrm{D}$ reconstruction of the renal artery. This may have led to an underestimation of the real prevalence and significance of ARVD. Another limitation of this study was that the LOCM iobitridol, iopromide, and iomeprol were analyzed together, assuming a comparable effect on outcome.

\section{Conclusions}

In high cardiovascular risk patients undergoing coronary angiography for ischemic heart disease, RVD was a stronger independent predictor of CIN than CKD.

\section{Acknowledgements}

Z.L. was supported by a European Renal Association-European Dialysis and Transplant Association (ERA-EDTA) long-term fellowship (ERALF 48-2009). 


\begin{tabular}{l|l}
\hline \begin{tabular}{l} 
Nephron Extra 2011;1:38-44 \\
\hline DOI: 10.1159/000329895
\end{tabular} & $\begin{array}{l}\text { @ 2011 S. Karger AG, Basel } \\
\text { www.karger.com/nne }\end{array}$ \\
\hline Published online: August 16, 2011 &
\end{tabular}

\section{Disclosure Statement}

The authors declare no conflicts of interest.

\section{References}

1 Nash K, Hafeez A, Hou S: Hospital-acquired renal insufficiency. Am J Kidney Dis 2002;39:930-936.

2 Rihal CS, Textor SC, Grill DE, Berger PB, Ting HH, Best PJ, Singh M, Bell MR, Barsness GW, Mathew V, Garratt KN, Holmes DR Jr: Incidence and prognostic importance of acute renal failure after percutaneous coronary intervention. Circulation 2002;105:2259-2264.

3 Senoo T, Motohiro M, Kamihata H, Yamamoto S, Isono T, Manabe K, Sakuma T, Yoshida S, Sutani Y, Iwasaka T: Contrast-induced nephropathy in patients undergoing emergency percutaneous coronary intervention for acute coronary syndrome. Am J Cardiol 2010;105:624-628.

4 McCullough PA, Wolyn R, Rocher LL, Levin RN, O’Neill WW: Acute renal failure after coronary intervention: incidence, risk factors, and relationship to mortality. Am J Med 1997;103:368-375.

5 Zanoli L, Rastelli S, Marcantoni C, Tripepi G, Matalone M, Tamburino C, Zoccali C, Castellino P: The renal artery calibre (RAC): a new insite in renal artery stenosis (Abstract). JASN 2008;19:839A840 A.

6 Marcantoni C, Zanoli L, Rastelli S, Tripepi G, Matalone M, Di Landro D, Scandura S, Tamburino C, Zoccali C, Castellino P: Stenting of Renal Artery Stenosis in Coronary Artery Disease (RAS-CAD) study: a prospective, randomized trial. J Nephrol 2009;22:13-16.

7 Marcantoni C, Rastelli S, Zanoli L, Tripepi G, Di Salvo M, Monaco S, Sgroi C, Capodanno D, Tamburino C, Castellino P: Prevalence of renal artery stenosis in patients undergoing cardiac catheterization. Intern Emerg Med 2011, June 26, Epub ahead of print.

8 Levey AS, Greene T, Kusek JW, Beck GJ: A simplified equation to predict glomerular filtration rate from serum creatinine (Abstract). J Am Soc Nephrol 2000;11:A0828.

9 McCullough PA: Beyond serum creatinine: defining the patient with renal insufficiency and why? Rev Cardiovasc Med 2003;4(suppl 1):S2-S6.

10 Davidson C, Stacul F, McCullough PA, et al: Contrast medium use. Am J Cardiol 2006;98:42K-58K.

11 Langille BL: Remodelling of developing and mature arteries: endothelium, smooth muscle and matrix. J Cardiovasc Pharmacol 1993;21:S11-S17.

12 Langille BL, O'Donnell F: Reductions in arterial diameter produced by chronic decreases in blood flow are endothelium-dependent. Science 1986;231:405-407.

13 Morcos SK, Thomsen HS, Webb JA: Contrast-media-induced nephrotoxicity: a consensus report. Eur Radiol 1999;9:1602-1613.

14 Katzberg RW: Urography into the 21st century: new contrast media, renal handling, imaging characteristics, and nephrotoxicity. Radiology 1997;204:297-312.

15 Kay J, Chow WH, Chan TM, et al: Acetylcysteine for prevention of acute deterioration of renal function following elective coronary angiography and intervention: a randomized controlled trial. JAMA 2003;289:553-558.

16 Kelly AM, Dwamena B, Cronin P, Bernstein SJ, Carlos RC: Meta-analysis: effectiveness of drugs for preventing contrast-induced nephropathy. Ann Intern Med 2008;148:284-294.

17 Briguori C, Colombo A, Violante A, et al: Standard versus double dose of $\mathrm{N}$-acetylcysteine to prevent contrast agent associated nephrotoxicity. Eur Heart J 2004;25:206-211. 